Communications in Physics, Vol. 24, No. 4 (2014), pp. 381-389

DOI:10.15625/0868-3166/24/4/5553

\title{
THE STUDY OF ISOMERIC RATIOS IN PHOTONUCLEAR REACTIONS FORMING HIGH SPIN ISOMERS IN THE GIANT DIPOLE RESONANCE REGION
}

\author{
TRAN DUC THIEP, TRUONG THI AN, PHAN VIET CUONG, NGUYEN THE VINH, \\ AND BUI MINH HUE \\ Institute of Physics, VAST, 10 Dao Tan St., Ba Dinh Region, Hanoi, Vietnam
}

A. G. BELOV AND O. D. MASLOV

Flerov Laboratory of Nuclear Reactions, JINR, 141980 Dubna, Moscow Region, Russia

E-mail: tdthiep@iop.vast.ac.vn

Received 26 November 2014

Accepted for publication 12 December 2014

\begin{abstract}
We studied the isomeric ratios in odd-odd nuclei ${ }^{196} \mathrm{Au},{ }^{182} \mathrm{Ta}$ and ${ }^{194}$ Ir with high spin isomeric states produced in ${ }^{197} \mathrm{Au}(\gamma, n){ }^{196 m, g} \mathrm{Au},{ }^{183} \mathrm{~W}(\gamma, p)^{182 m, g}$ Ta and ${ }^{185} \mathrm{Pt}(\gamma, p)^{194 m, g}$ Ir reactions by using the activation technique and $\gamma$-ray spectroscopic method in the giant dipole resonance $(G D R)$ region. The high-purity natural Au, W and Pt foils in disc shape were irradiated with bremsstrahlungs generated from an electron accelerator Microtron. The irradiated foils were measured by the high resolution $\gamma$-ray spectroscopic system which consists of a Ge(HP) detector and a multichannel analyzer. In order to improve the accuracy of the experimental results, necessary corrections were made in the $\gamma$-ray activity measurements and data analysis. The results were analyzed, discussed and compared with those of other authors as well as with theoretical model calculations. The study shows that the isomeric ratios in nuclei with high spin isomeric states are much lower than that in low spin isomeric state isomers.
\end{abstract}

Keywords: photonuclear reaction, isomeric ratio, GDR region, reaction yield-impulse.

\section{INTRODUCTION}

The isomeric ratios furnish valuable information about the energy level structure of nuclei and the nuclear reaction mechanism involved [1-5]. This quantity depends on the spin of target nucleus and the intake angular momentum determined by the mass and the energy of projectile particles. By fitting the isomeric ratios calculated on the basis of a definite theoretical model to the experimental ones, it is possible to obtain information about the spin dependence of the nuclear level density, in particular, the spin cut-off parameter $\sigma$ and the level density parameter $a$. The investigation of isomeric ratio in photonuclear reactions with bremsstrahlung has some advantages although the cross section of photonuclear reaction is very low due to the reasons: bremsstrahlungs are an intense source of photon produced by high power electron accelerators and the cross section of photonuclear reaction presents a wide resonance peak. Consequently, the reaction yield is significant. In addition, the study on the isomeric ratio in photonuclear reaction 
are also very interest due to the significant difference from nuclear reaction induced by other projectiles. This difference is connected to the fact that in photonuclear reaction other isomeric states are excited which are usually difficult to be produced in other reaction types.

In photonuclear reactions in the giant dipole resonance (GDR) region, the absorption of an $E 1 \gamma$-ray leads the target nucleus with spin $J_{0}$ to an excited compound nucleus with spins $J_{0} \pm 1$. One of the important properties of photonuclear reactions in the GDR region is that their crosssections are characterized with a wide maximum, so called "giant resonance" and the most probable mechanism of interactions is the process of successive emission of one or several nucleons from the compound nucleus. In this region the electromagnetic interaction is well known and absorption of $E 1 \gamma$-ray is dominant, therefore the theoretical consideration will be simplified. In the case of photonuclear reactions with bremsstrahlung, the isomeric ratio is defined as the ratio of the production yield of (or the probability of forming) the isomeric state and that of the ground state. On the other hand, besides the spectrum of excited states, level and energy densities, multipolity, the probability of forming a state strongly depends on its spin. Therefore, the isomeric ratio will depend on the spins of the isomeric and ground states as well as on their difference.

The aim of this work is to study the isomeric ratios in nuclei in region of $Z=73-79$ and $A=182-196$. In this region, the nuclear levels with high angular momentum $\left(h_{11 / 2}\right.$ proton and $i_{13 / 2}$ neutron shell) are populated and as a reason the odd-odd nuclei with high spin isomers will be appeared. As nuclei under investigation were chosen ${ }^{196} \mathrm{Au},{ }^{182} \mathrm{Ta}$ and ${ }^{184} \mathrm{Ir}$ which can be produced via $(\gamma, \mathrm{n})$ and $(\gamma, \mathrm{p})$ reactions and their isomeric states have spin higher than $10 \hbar$.

\section{EXPERIMENTAL}

\section{Target preparation}

The samples for investigation were prepared from natural gold, tungsten and platinum foils with purity of $99.99 \%$ in disk shape $1 \mathrm{~cm}$ in diameter with masses of $0.215,0.320$ and 0.250 $\mathrm{g}$ respectively. Table 1 shows the decay characteristics of isomeric pairs ${ }^{182 m, g} \mathrm{Ta},{ }^{196 m, g} \mathrm{Au}$ and ${ }^{194 m, g}$ Ir which have been observed in the experiments with bremsstrahlungs in the GDR region. The data were taken from $[6,7]$.

\section{Target irradiation}

The sample irradiation in the GDR region was performed at the electron accelerator $\mathrm{Mi}$ crotron MT-25 of the Flerov Laboratory of Nuclear Reaction, Joint Institute for Nuclear Research Dubna, Russia. The technical details of this accelerator are presented in [8]. The essential advantages of this Microtron are the small energy spread of the accelerated electrons $(30-40 \mathrm{keV})$ at high beam intensity (up to an average power of $600 \mathrm{~W}$ ). This allows the measurement of the isomeric ratio at strictly definite end-point energy bremsstrahlung. As an electron-photon converter was used $\mathrm{W}$ disk $0.3 \mathrm{~mm}$ in thickness, cooled by water. To absorb low energy electrons passing the converter in the irradiation target, an Aluminum screen $20 \mathrm{~mm}$ in thickness was placed behind the converter. The end- point bremsstrahlung energy of this accelerator can be varied stepwise from 10 to $25 \mathrm{MeV}$ i.e. the GDR region. Four gold samples were irradiated with with end-point bremsstrahlung energies $15,18,21.5$ and $24.0 \mathrm{MeV}$ and the average electron beam current was about $12 \mu \mathrm{A}$. For wolfram two samples were irradiated with 15.0 and $21.5 \mathrm{MeV}$ bremsstrahlungs and the average electron beam current was about $15 \mu \mathrm{A}$. Two samples of platinum were irradiated with 19.0 and $24.0 \mathrm{MeV}$ bremsstrahlungs and the average electron beam current was about $14 \mu \mathrm{A}$. 
Table 1. The decay characteristics of nuclei under investigation and gamma rays used

\begin{tabular}{|c|c|c|c|c|c|c|c|}
\hline $\begin{array}{c}\text { Nuclear Reaction } \\
\text { and } \\
\text { Target spin }\end{array}$ & $\begin{array}{l}\text { Reaction } \\
\text { Product }\end{array}$ & $\begin{array}{l}\text { Spin } \\
{[\hbar]}\end{array}$ & $\begin{array}{c}\text { Abun. } \\
{[\%]}\end{array}$ & Half life & $\begin{array}{c}\text { Reaction } \\
\text { Threshold } \\
{[\mathrm{MeV}]}\end{array}$ & $\begin{array}{c}\text { Gamma ray } \\
\text { Energy [KeV] } \\
\text { and } \\
\text { Intensity [\%] }\end{array}$ & $\begin{array}{l}\text { Isom. } \\
\text { Trans. } \\
\text { coeff. } \\
\text { IT [\%] }\end{array}$ \\
\hline $\begin{array}{c}{ }^{183} \mathrm{~W}(\gamma, \mathrm{p})^{182 m, g} \mathrm{Ta} \\
I=1 / 2^{-}\end{array}$ & $\begin{array}{l}{ }^{182 m} \mathrm{Ta} \\
{ }^{182 g} \mathrm{Ta}\end{array}$ & $\begin{array}{l}10^{-} \\
3^{-}\end{array}$ & 14.4 & $\begin{array}{l}15.8 \mathrm{~min} \\
115 \mathrm{~d}\end{array}$ & 7.21 & $\begin{array}{c}146.8(29.6) \\
171.7(47.0) \\
184.9(23.5) \\
100.0(14.4) \\
1121.0(37.0) \\
1188.0(16.5) \\
1221.0(27.4)\end{array}$ & 100.0 \\
\hline $\begin{array}{c}{ }^{197} \mathrm{Au}(\gamma, n){ }^{196 m, g} \mathrm{Au} \\
I=3 / 2^{+}\end{array}$ & $\begin{array}{l}{ }^{196 m} \mathrm{Au} \\
{ }^{196 g} \mathrm{Au}\end{array}$ & $\begin{array}{l}12^{-} \\
2^{-}\end{array}$ & 100 & $\begin{array}{l}9.7 \mathrm{~h} \\
6.183 \mathrm{~d}\end{array}$ & $\begin{array}{l}8.68 \\
8.09\end{array}$ & $\begin{array}{l}147.7(43.00) \\
188.3(37.84) \\
333.0(27.15) \\
355.0(87.60)\end{array}$ & 100.0 \\
\hline $\begin{array}{c}{ }^{195} \mathrm{Pt}(\gamma, \mathrm{p}){ }^{194 m, g} \operatorname{Ir} \\
\mathrm{I}=1 / 2^{-}\end{array}$ & $\begin{array}{l}{ }^{194 m} \mathrm{Ir} \\
{ }^{194 g} \mathrm{Ir}\end{array}$ & $\begin{array}{c}11^{+} \\
1^{-} \\
\end{array}$ & 33.8 & $\begin{array}{c}171.55 \mathrm{~d} \\
19.15 \mathrm{~h}\end{array}$ & $\begin{array}{l}7.58 \\
7.58 \\
\end{array}$ & $\begin{array}{l}390.8(35.00) \\
293.5(2.52)\end{array}$ & 0.0 \\
\hline
\end{tabular}

\section{Gamma spectra measurement}

The irradiated samples were measured for different cooling and measurement times with a spectroscopic system consisting of 8192 channel analyzer and high-energy resolution $(180 \mathrm{keV}$ at gamma ray $1332 \mathrm{keV}$ of ${ }^{60} \mathrm{Co}$ ) $\mathrm{HP}(\mathrm{Ge})$ semiconductor detector Canberra. The GENIE2000 (Canberra) computer program was used for data processing. The efficiency curve of the detector were determined with a set of standard single gamma ray sources calibrated to 1- $2 \%$.

\section{Isomeric ratio determination}

The isomeric ratio is defined as the ratio of the production yield of the isomeric state to that of the ground state. In the case of bremsstrahlung it can be expressed as follows:

$$
I R=\frac{N o \int_{E_{t h}^{m}}^{E_{\gamma}^{m}} \sigma_{m}(E) \phi(E) d E}{N o \int_{E_{t h}^{g}}^{E_{\gamma}^{m}} \sigma_{g}(E) \phi(E) d E}
$$

Usually the isomeric ratio in a nuclear reaction is determined by the measurements of the areas under gamma rays characterizing the isomeric and the ground states. The equations that describe the decay of these states can be written as follows:

$$
\begin{aligned}
\frac{d N_{m}}{d t} & =N o \phi \sigma_{m}-\lambda_{m} N_{m}, \\
\frac{d N_{g}}{d t} & =N o \phi \sigma_{g}-\lambda_{g} N_{g}+P \lambda_{m} N_{m},
\end{aligned}
$$


By resolving system of equations (2) in dependence on irradiating, cooling and measurement times we could determine the isomeric ratio $I R$ by the expression below:

$$
\frac{1}{I R}=\frac{\frac{S_{g} \varepsilon_{m} I_{m}}{S_{m} \varepsilon_{g} I_{g}} \Lambda_{3} \Lambda_{6} \Lambda_{9}-\Lambda_{1} \Lambda_{5} \Lambda_{8}-\Lambda_{3} \Lambda_{5} \Lambda_{8}-\Lambda_{3} \Lambda_{6} \Lambda_{7}}{\Lambda_{2} \Lambda_{5} \Lambda_{8}}
$$

where the index of $m$ and $g$ denote the isomeric and ground states; No- the target nuclei number; $\phi(E)$ - bremsstrahlung photon flux; $N$ - the number of reaction product nuclei; $\lambda$ - the decay constant and $P$-the isomeric transition coefficient; $S, \varepsilon$ and $I$ - the areas, the efficiencies and the intensities of the interested gamma rays; $E_{\gamma}^{m}$ - the bremsstrahlung maximum energy, $\sigma_{m}(E)$ and $\sigma_{g}(E)$ - the cross sections of the isomeric and ground states respectively; $E_{t h}^{m}$ and $E_{t h}^{g}$ - the threshold energies for the isomeric and ground states respectively and $\Lambda_{i}(i=1 \sim 9)$ are expressions connected to the irradiating, cooling and measurement times.

\section{Theoretical model calculation}

In this work, the statistical model proposed by Huizenga and Vandenbosch [1,2] was applied for calculation of the isomeric ratio. Though this model has been established long time ago, however it is still very powerful and widely used tool for nuclear reaction investigations. In the Huizenga - Vandenbosch model, the theoretical isomeric ratios are calculated as follows:

$$
R=\frac{\sum_{J_{f}=\cos }^{\infty} P\left(J_{f}\right)}{\sum_{J_{f}=\cos }^{M} P\left(J_{f}\right)}
$$

where $C O S=\left(S_{h}+S_{l}\right) / 2 ; S_{h}$ and $S_{l}$ - spins of high and low spin states; $P(J)$ - the probability for forming an ensemble of states with different final spin $J$ after the successive evaporation of one neutron from compound nucleus. reactions:

We begin by considering the following steps for special case of $(\gamma, n)$ or $(\gamma, p)$ photonuclear

Absorption of an E1 $\gamma$-ray brings a target nucleus with spin $J_{o}$ to forming an excited compound nucleus with the relative occupation probability:

$$
P\left(J_{c}\right) \simeq 2 J_{c}+1, J_{c}=J_{0}, J_{0}-1, J_{0}+1
$$

Relative probability for forming a residual nucleus with spin $\mathrm{J}$ after the successive evaporation of one neutron or one proton is:

$$
P(J) \approx \rho\left(E^{*}, J\right) \sum_{S=|J-1 / 2|}^{J+1 / 2} \sum_{J_{c}} P\left(J_{c}\right) \sum_{l=\left|S-J_{c}\right|}^{S+J_{c}} T_{l}\left(E_{n}\right)
$$

where $T_{l}\left(E_{n}\right)$ - the penetrability for a neutron or a proton with angular momentum $l$ and kinetic energy $E_{n} ; \rho\left(E^{*}, J\right)$ - the level density of excited nucleus, represented by the following formula:

$$
\rho\left(E^{*}, J\right)=\rho\left(E^{*}\right) \rho(J) \sim \exp \left[2\left(a E^{*}\right)^{\frac{1}{2}}\right](2 J+1) \exp \left[-\frac{J(J+1)}{2 \sigma^{2}}\right]
$$

where $E^{*}$ the excitation energy of residual nucleus; $\sigma$ the spin cut-off parameter and $a$ - the level density parameter. 
In calculation we supposed that the energy distribution of emitted neutron or proton has Maxwell form and the average kinetic energy is:

$$
\overline{E_{n}}=\frac{1+\sqrt{1+4 a U}}{2 a}
$$

where $U$ - the excitation energy of compound nucleus, which is reated to the separation and kinetic energies ofemitted neutron or proton and the excitation energy of the residual nucleus by the following expression:

$$
E^{*}=U-S_{n}-E_{n}
$$

where $S_{n}$ and $E_{n}$ - the separation and kinetic energies of emitted neutron or proton.

If $\mathrm{E}^{*}$ is below the particle threshold it is supposed that the residual nucleus de-excites predominantly by E1 gamma-ray emission with an average energy for $(l+1)$ th gamma-ray:

$$
E_{\gamma(i+1)}=4\left[\frac{E^{*}-\sum^{E_{\gamma i}}}{a}-\frac{5}{a^{2}}\right]^{1 / 2}
$$

Here it is supposed that $\mathrm{E}_{\gamma 0}=0$.

The gamma cascade continues until the residual energy, i.e. $E=E^{*}-\sum E_{\gamma i}$ is smaller than "the $\gamma$-ray cut-off region" $\left(E_{u}, E_{d}\right)$; then the following $\gamma$-ray, the so-called "the deciding $\gamma$-ray" is emitted, feeds that states to which the transition has the lowest multipolity. The gamma ray transition probability from states with spin $\mathrm{J}_{i}$ to those with spin $J_{f}$ is assumed to be:

$$
P\left(J_{f}\right) \approx \sum P\left(J_{i}\right) \rho\left(J_{f}\right)
$$

The residual energy remains within "the $\gamma$-ray cut-off region", partly a subsequent E1 $\gamma$ ray is emitted and partly "the deciding $\gamma$-ray" is emitted as described above. In this case the competing channel with transition probabilty $P=\left(E-E_{d}\right) /\left(E_{u}-E_{d}\right)$ must be taken into account and the isomeric ratios will be calculated by the following formula:

$$
R=P \frac{\sum_{J_{f}=0}^{\cos } P\left(J_{f}, E-E_{d}\right)}{\sum_{J_{f}=\cos }^{\infty} P\left(J_{f}, E-E_{d}\right)}+(1-P) \frac{\sum_{J_{f}=0}^{\cos } P\left(J_{f}, E\right)}{\sum_{J_{f}=\cos }^{\infty} P\left(J_{f}, E\right)}
$$

In the case of bremsstrahlung induced photonuclear reactions, the isomeric ratios are calculated as the average value:

$$
\bar{R}=\frac{\int_{E_{t h}}^{E_{m}} \phi\left(E_{\gamma}\right) \sigma(\gamma, n) R d E}{\int_{E_{t h}}^{E_{m}} \phi\left(E_{\gamma}\right) \sigma(\gamma, n) d E}
$$

where $E_{t h} ; E_{m}$ and $\phi(E)$ - the threshold energy, the bremsstrahlung maximum energy and the bremsstrahlung photon flux respectively, and $\sigma(\gamma, n)$ - the excitation function or cross section of $(\gamma, n)$ reaction. The data for $\sigma(\gamma, n)$ were taken from [21] and for $\phi(E)$ by Shiff approximation [23] or by simulation method [24].

More detailed description of the program for theoretical model calculation of the isomeric ratio using Huizenga and Vandenbosch method can be found in [22]. 


\section{RESULTS AND DISCUSSION}

For demonstration of the experimental procedure in Fig. 1 is shown a typical gamma spectrum of natural platinum irradiated by $24.0 \mathrm{MeV}$ bremsstrahlung measured with the $\mathrm{HP}(\mathrm{Ge})$ semiconductor detector at a distance of $5 \mathrm{~cm}$. The gamma rays characterizing the isomeric and ground states of isomeric pair ${ }^{194 m, g} \mathrm{Ir}$ are marked on this spectrum. Other gamma rays were arisen from different products of the interaction between natural platinum and the bremsstrahlung. The isomeric ratio was determined by using expression (3) as the average value $\overline{I R}$ of those data calculated from various combinations of a series of gamma spectra measured for different times of cooling and measurement. The relative error was determined by the following formula:

$$
\varepsilon(\overline{I R})=\frac{\sigma(\overline{I R})}{\overline{I R}}=\frac{\sqrt{\sum_{i=1}^{n} \sigma_{i}^{2}(I R) / n}}{\overline{I R}},
$$

where $\sigma_{i}(I R)$ - the error of the isomeric ratio calculated for $i$ - combination of measurements and $n$ - the number of combinations of the measurement.

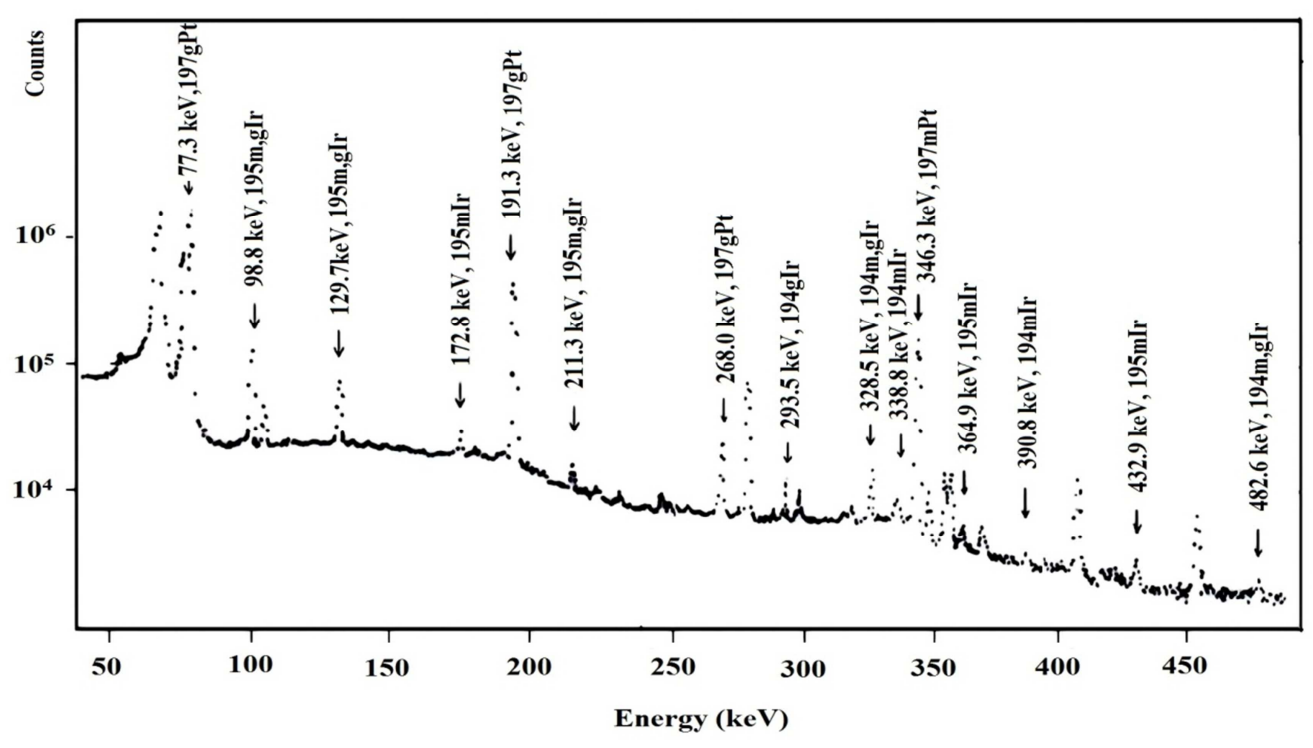

Fig. 1. A typical gamma spectrum of natural Pt irradiated by $24.0 \mathrm{MeV}$ end-point bremsstrahlung energy.

In the experiment, the irradiation, cooling and measurement times were properly chosen to reach the statistical errors of radioactive measurement less than $0.1 \%, 3 \%$ and $4.5 \%$ for nuclei ${ }^{196} \mathrm{Au},{ }^{182} \mathrm{Ta}$ and ${ }^{194} \mathrm{Ir}$ respectively. The counting loss was arisen from coincidence summing and gamma ray self- absorption. The corrections for these effects were performed in the experiment by the methods used in $[19,20]$ based on the efficiencies of summing gamma rays and attenuation coefficient. By taking proper distance from sample to detector and optimum sample mass, the 
counting losses were estimated less than $0.5 \%$ i.e much insignificant in comparison with other errors. In fact by this way it was not seen coincidence summing gamma ray in the gamma spectrum. In order to reduce the count loss due to dead time and file-up, especially at the end of irradiation there are methods existed. In practice for the advanced gamma spectroscopy system, the dead time is always shown during measurement and the correction can be made. However in some experiments the dead time is too high and count loss is significant. In this case the dead time can be reduced as follows: a/ If the half-lives of the isomeric and ground states are enough long then (as in case of ${ }^{196 m, g} \mathrm{Au}$ and ${ }^{194 m, g}$ Ir shown in Table 1), the measurement will be started after properly long cooling time to reduce the dead time, $\mathrm{b}$ / If the half-lives of the isomeric and ground states are enough short (as in case of ${ }^{182 m}$ Ta shown in Table 1), the measurement has to be started as soon as the irradiation stops, then the dead time can be reduce by taking proper distance between sample and detector. It is worth noting that when the dead time is proper value and the measurement is in the live-time regime then the dead time correction is not needed in calculation of the experimental isomeric ratio. The total errors of the isomeric ratios are 5\%,10\% and $13 \%$ for nuclei ${ }^{196} \mathrm{Au}$, ${ }^{182} \mathrm{Ta}$ and ${ }^{194}$ Ir respectively. Table 2 presents the experimental results and calculated ones of the isomeric ratios under investigation. One can see the following facts:

Table 2. The experimental results and calculated ones of the isomeric ratios under investigation.

\begin{tabular}{|c|c|c|c|c|c|c|}
\hline \multirow{2}{*}{$\begin{array}{l}\text { End- } \\
\text { point } \\
\text { Energy } \\
{[\mathrm{MeV}]}\end{array}$} & \multicolumn{2}{|c|}{${ }^{197} \mathrm{Au}(\gamma, \mathrm{n}){ }^{196 m, g} \mathrm{Au}$} & \multicolumn{2}{|c|}{${ }^{183} \mathrm{~W}(\gamma, \mathrm{p}){ }^{182 m, g} \mathrm{Ta}$} & \multicolumn{2}{|c|}{${ }^{195} \operatorname{Pt}(\gamma, \mathrm{p}){ }^{194 m, g} \operatorname{Ir}$} \\
\hline & Experimental & Calculated & Experimental & Calculated & Experimental & Calculated \\
\hline 12.0 & 1. $10^{-4}[9]$ & & & & & \\
\hline 14.0 & 5. $10^{-4}[9]$ & & & & & \\
\hline 15.0 & 1.28. $10^{-4}\left[{ }^{*}\right]$ & 1.30. $10^{-4}\left[{ }^{*}\right]$ & 7.7. $10^{-4}[*]$ & & & \\
\hline 16.0 & $1.20 \cdot 10^{-3}[9]$ & & & & & \\
\hline 18.0 & 2.94. $10^{-4}[*]$ & & & & & \\
\hline 19.0 & & & & & 2.5. $10^{-4}[*]$ & \\
\hline 21.5 & 3.78. $10^{-4}[*]$ & 4.10. $10^{-4}[*]$ & 8.1. $10^{-4}\left[{ }^{*}\right]$ & & & \\
\hline 22.0 & $6.1 .10^{-4}[13]$ & & & & & \\
\hline 24.0 & $\begin{array}{l}\text { 4.03.1 } 10^{-4}[*] \\
\text { 3.0. } 10^{-3}[9]\end{array}$ & 4.15. $10^{-4}[*]$ & & & 2.9. $10^{-4}[*]$ & \\
\hline & 3.1. $10^{-4}[12]$ & 8. $10^{-5}[12]$ & 2.2. $10^{-4}[12]$ & 2. $10^{-4}[12]$ & $<10^{-3}[12]$ & 8. $10^{-4}[12]$ \\
\hline 50.0 & $\begin{array}{l}\text { 5. } 10^{-4}[10] \\
\text { 6. } 10^{-4}[9]\end{array}$ & & & & & \\
\hline 52.0 & 5. $10^{-4}[11]$ & & & & & \\
\hline
\end{tabular}

[*] This work

Up to now there has been very few works devoted to the study of isomeric ratios in photonuclear reactions forming high spin isomers in the GDR region as well as in region of higher energies. In general our results and the data in [10 - 13] are in good agreement and much lower than that in [9] for the energy region from 15 to $24.0 \mathrm{MeV}$. However we can make a comment on 
the data in [9] when referring to the excitation function of ${ }^{197} \mathrm{Au}(\gamma, \mathrm{n}){ }^{196} \mathrm{Au}$ reaction presented in [21]. At $14 \mathrm{MeV}$ the integrated cross-section is about 1,200 MeV.mb and at $18 \mathrm{MeV}$ this value is about 2,400 MeV.mb i.e. the integrated increases 2 times while the isomeric ratio increases 4.6 times. It seems that the data in [9] may have to be reconsidered.

Table 3. The isomeric ratios in some product nuclei produced from photonuclear reactions at $23.5 \mathrm{MeV}$ bremsstrahlung

\begin{tabular}{|l|l|l|l|l|l|l|}
\hline $\begin{array}{l}\text { Nuclear } \\
\text { Reaction }\end{array}$ & $\begin{array}{l}\text { Product } \\
\text { nucleus }\end{array}$ & $\begin{array}{l}\text { High } \\
\text { spin }\end{array}$ & $\begin{array}{l}\text { Low } \\
\text { spin }\end{array}$ & $\begin{array}{l}\text { Spin } \\
\text { difference }\end{array}$ & $\begin{array}{l}\text { Isomeric } \\
\text { ratio }\end{array}$ & Reference \\
\hline${ }^{121} \mathrm{Sb}(\gamma, \mathrm{n})^{120 m, g} \mathrm{Sb}$ & ${ }^{120} \mathrm{Sb}$ & $8^{-}$ & $1^{+}$ & 7 & 0.0516 & {$[14]$} \\
${ }^{123} \mathrm{Sb}(\gamma, \mathrm{n})^{122 m, g} \mathrm{Sb}$ & ${ }^{122} \mathrm{Sb}$ & $8^{-}$ & $2^{+}$ & 6 & 0.0154 & {$[14]$} \\
${ }^{142} \mathrm{Nd}(\gamma, \mathrm{n})^{141 m, g} \mathrm{Nd}$ & ${ }^{141} \mathrm{Nd}$ & $11 / 2^{-}$ & $3 / 2^{+}$ & $8 / 2$ & 0.049 & {$[15]$} \\
${ }^{110} \mathrm{Pd}(\gamma, \mathrm{n})^{109 m, g} \mathrm{Pd}$ & ${ }^{109} \mathrm{Pd}$ & $11 / 2^{-}$ & $5 / 2^{+}$ & $6 / 2$ & 0.068 & {$[15]$} \\
${ }^{74} \mathrm{Se}(\gamma, \mathrm{n})^{73 m, g} \mathrm{Se}$ & ${ }^{73} \mathrm{Se}$ & $9 / 2^{+}$ & $3 / 2^{-}$ & $6 / 2$ & 0.72 & {$[16]$} \\
${ }^{82} \mathrm{Se}(\gamma, \mathrm{n})^{81 m, g} \mathrm{Se}$ & ${ }^{81} \mathrm{Se}$ & $7 / 2^{+}$ & $1 / 2^{-}$ & $6 / 2$ & 0.54 & {$[16]$} \\
${ }^{175} \mathrm{Lu}(\gamma, \mathrm{n})^{174 m, g} \mathrm{Lu}$ & ${ }^{174} \mathrm{Lu}$ & $6^{-}$ & $1^{-}$ & 5 & 0.452 & {$[17]$} \\
${ }^{197} \mathrm{Au}(\gamma, \mathrm{n})^{196 m, g} \mathrm{Au}$ & ${ }^{196} \mathrm{Au}$ & $12^{-}$ & $2^{-}$ & 10 & $4.03 .10^{-4}$ & This work \\
${ }^{195} \mathrm{Pt}(\gamma, \mathrm{p})^{194 m, g} \mathrm{Ir}$ & ${ }^{194} \mathrm{Ir}$ & $11^{+}$ & $1^{-}$ & 10 & $2.9 .10^{-4}$ & This work \\
${ }^{183} \mathrm{~W}(\gamma, \mathrm{p})^{182 m, g} \mathrm{Ta}$ & ${ }^{182} \mathrm{Ta}$ & $10^{-}$ & $3^{-}$ & 7 & $2.2 .10^{-4}$ & {$[12]$} \\
${ }^{191} \mathrm{Ir}(\gamma, \mathrm{n})^{190 m, g} \mathrm{Ir}$ & ${ }^{190} \mathrm{Ir}$ & $11^{-}$ & $4^{-}$ & 7 & $8.10^{-4}$ & {$[18]$} \\
${ }^{207} \mathrm{~Pb}(\gamma, \mathrm{p})^{206 m, g} \mathrm{Tl}$ & ${ }^{206} \mathrm{Tl}$ & $12^{-}$ & $0^{-}$ & 12 & $2.4 .10^{-5}$ & {$[12]$} \\
\hline
\end{tabular}

In all odd-odd nuclei ${ }^{196} \mathrm{Au},{ }^{182} \mathrm{Ta}$ and ${ }^{194}$ Ir having high spin isomeric states, the isomeric ratios are extraordinarily low in comparison to other nuclei with lower spin isomeric state. This phenomenon can be clearly seen in Table 3 which shows the isomeric ratios in some product nuclei produced from photonuclear reactions at $24.0 \mathrm{MeV}$ bremsstrahlung. This fact can be related to the special properties and structure of the mentioned nuclei as they are nuclei in the transition region from strongly deformed nucleus ${ }^{182} \mathrm{Ta}$ to spherical nucleus ${ }^{206} \mathrm{Tl}$. Here it is interesting to note that the isomeric ratios also depend on the difference between spins of the isomeric and ground states and in general the higher the difference the lower the isomeric ratio.

From Table 2 one can see that for three nuclei ${ }^{196} \mathrm{Au},{ }^{182} \mathrm{Ta}$ and ${ }^{194} \mathrm{Ir}$ the isomeric ratios increase with the increase of end-point bremsstrahlung energies. This means that the probability for population of high spin state increases faster than that of low spin one when end-point bremsstrahlung energy increases.

The statistical model proposed by Huizenga and Vandenbosch used in this work is based on separation of a nuclear reaction in three stages as follows: a/ Formation of compound nucleus by absorption an E1 $\gamma$-ray, b/ Emission of neutron or proton from the excited compound nucleus and formation of residual nucleus and c/ Emission of $\gamma$-ray cascades from the residual nucleus leads to population of the isomeric and ground states. One can see from Table 2 that for ${ }^{196 m, g} \mathrm{Au}$ our experimental results and calculated ones are in good agreement and for ${ }^{182 m, g} \mathrm{Ta}$ and ${ }^{194 m, g} \mathrm{Ir}$ the experimental and calculated data of authors in [12] are in good agreement. This means that the statistical model is applicable for consideration of photonuclear reactions in the GDR region. 


\section{CONCLUSIONS}

The isomeric ratios in odd-odd nuclei of high spin isomers ${ }^{196} \mathrm{Au},{ }^{182} \mathrm{Ta}$ and ${ }^{194} \mathrm{Ir}$ produced in ${ }^{197} \mathrm{Au}(\gamma, \mathrm{n}){ }^{196 m, g} \mathrm{Au},{ }^{183} \mathrm{~W}(\gamma, \mathrm{p}){ }^{182 m, g} \mathrm{Ta}$ and ${ }^{195} \mathrm{Pt}(\gamma, \mathrm{p}){ }^{194 m, g}$ Ir reactions in GDR region have been studied. The obtained results show that the isomeric ratios in the mentioned nuclei are extraordinarily low and they could be used to check nuclear reaction models. The results could also contribute to the Nuclear Data.

\section{ACKNOWLEDGEMENTS}

This work has been performed at the Flerov Laboratory of Nuclear Reaction, Joint Institute for Nuclear Research, Dubna, Russia. The authors would like to express sincere thanks to the Chemical Department of the Flerov Laboratory of Nuclear Reaction for providing the measurement system.

This research is funded by Vietnam National Foundation for Science and Technology Development (NAFOSTED) under grant number 103.04-2012.56.

\section{REFERENCES}

[1] J. R. Huizenga, R. Vandenbosch, Phys. Rev. 120 (1960)1306

[2] J. R. Huizenga, R. Vandenbosch, Phys. Rev. 120 (1960)1313

[3] H. Bartsch, K. Huber, U. Kneissl, H. Krieger Nucl. Phys. A N.256 (1976)243

[4] N. Tsoneva, Ch. Stoyanov, Yu. P. Gangrsky, V. Yu. Ponomarev, N. P. Balabanov, and A. P. Tonchev, Phys. Rev. C 61 (2000) 044303

[5] D. Kolev, J. Ernest, Phys G: Nucl. Part. Phys. N.24 (1998) 589. doi: 10.1088/0954-3899/24/3/010

[6] Firestone RB, Table of isotopes, CD ROM Edn, Version 1.0, Wiley - Interscience., New York 1996

[7] Radiation Search, http://ie.lbl.gov/toi/radSearch.asp

[8] A. G. Belov et al., Preprint of JINR D15-93-80, 1993 Dubna, Russia.

[9] L. Z. Djilavian et al., Phys. Atomic Nuclei 3(3) (1981) 519.

[10] A. A. Sorokin et al., Report of XXVII Conf. on Nuclear Spectroscopy and Structure of Atomic Nuclei, 1976, p.449 (in Russian).

[11] A. A. Sorokin et al, Report of XXVII Conf. on Nuclear Spectroscopy and Structure of Atomic Nuclei, 1978, p.258 (in Russian).

[12] A. G. Belov et al., Phys. Part. Nucl. Letters 1(4)(2004)47

[13] Iu. P. Gangrski et al., Iz. Acad. Nauk. Ser. Fiz. 68(2) (2004)167

[14] T. D. Thiep et al., Nucl. Phys. A 722(2003)568

[15] T.D. Thiep et al., Bulg. J. Phys. 14(2) (1987) 52.

[16] V. M. Mazur, I. V. Sokolyuk, Z. M. Bigan, J Phys. Atomic Nuclei 54 (1991) 541

[17] T.D. Thiep et al., Radioanal. Nucl. Chem. 290(2) (2011) 515

[18] Iu. Gangrski et al, Proceedings of 53th International Workshop on Nuclear Spectroscopy and Structure of Atomic Nuclei, Moscow 2003, p.184

[19] K. S. Kim, M. D. Shakilur Rahman, G. N. Kim GN et al., Radioanal. Nucl. Chem. 287 (2011) 869.

[20] N. V. Do, P. D. Khue, G. N. Kim et al, Radioanal. Nucl. Chem. 287(2011) 813.

[21] B. L. Berman, Atomic Nuclear Tables 15 (1975) 319

[22] T. D. Thiep, N. N. Son and N. T. Khai, Comm. in Phys. 7(1) (1977)21.

[23] L. I. Schiff, Phys. Rev. 83(1951) 253.

[24] T. D. Thiep, N. T. Khai, Comm. in Phys. 12(1) (2002) 48. 